\title{
A phase I study of farletuzumab, a humanized anti-folate receptor $\alpha$ monoclonal antibody, in patients with solid tumors
}

\author{
Yasutsuna Sasaki • Keisuke Miwa • Keishi Yamashita - Yu Sunakawa • Ken Shimada • Hiroo Ishida • \\ Kosei Hasegawa $\cdot$ Keiichi Fujiwara $\cdot$ Makoto Kodaira • Yasuhiro Fujiwara $\cdot$ Masayuki Namiki \\ Minami Matsuda $\cdot$ Yutaka Takeuchi $\cdot$ Noriyuki Katsumata
}

Received: 1 September 2014 / Accepted: 17 October 2014 / Published online: 9 November 2014

(C) The Author(s) 2014. This article is published with open access at Springerlink.com

Summary Farletuzumab is a humanized monoclonal antibody against folate receptor $\alpha$ (FRA). The purpose of the study is to assess safety and tolerability, the pharmacokinetic (PK) profile, and preliminary antitumor effect. Patients with ovarian cancer (OC) or FRA-expressing solid tumors who are resistant to standard treatments were eligible for the study. After single-dose administration for PK assessment, farletuzumab was administered by intravenous injection, repeating every week until disease progression. Dose-limiting

Presented in part at the 48th Annual Meeting of the American Society of Clinical Oncology, Chicago, IL, June 1-5, 2012.

Y. Sasaki $\cdot$ K. Miwa $\cdot$ K. Yamashita $\cdot$ Y. Sunakawa $\cdot$ K. Shimada $\cdot$

H. Ishida

Department of Medical Oncology, Saitama International Medical

Center-Comprehensive Cancer Center, Saitama Medical University,

Saitama, Japan

K. Hasegawa $\cdot$ K. Fujiwara

Department of Gynecologic Oncology, Saitama International

Medical Center-Comprehensive Cancer Center, Saitama Medical

University, Saitama, Japan

M. Kodaira $\cdot$ Y. Fujiwara $\cdot$ N. Katsumata

Department of Breast and Medical Oncology, National Cancer

Center Hospital, Tokyo, Japan

M. Namiki $\cdot$ M. Matsuda $\cdot$ Y. Takeuchi

Eisai Co., Ltd., Tokyo, Japan

Present Address:

Y. Sasaki $(\square) \cdot$ H. Ishida

Division of Medical Oncology, Department of Medicine, Showa

University, School of Medicine, 1-5-8 Hatanodai, Shinagawa-ku,

Tokyo 142-8666, Japan

e-mail: yasutsuna@med.showa-u.ac.jp toxicities (DLTs) were defined as grade 4 hematological and grade $3 / 4$ nonhematological toxicities. Dose escalation was planned in 4 cohorts $\left(50,100,200\right.$, and $\left.400 \mathrm{mg} / \mathrm{m}^{2}\right)$. Fourteen patients with $\mathrm{OC}$ and two patients with gastric cancer (GC) received farletuzumab infusion. Neither DLTs nor grade 3/4 toxicities were reported in all cohorts. Major adverse events, including grade $1 / 2$ infusion related reaction (15 patients, $93.8 \%$ ), headache (seven patients, $43.8 \%$ ), and nausea and decreased appetite (five patients each, $31.3 \%$ ), were observed

Present Address:

K. Miwa

Multidisciplinary Treatment Cancer Center, Kurume University

Hospital, Fukuoka, Japan

Present Address:

K. Yamashita

Kawagoe Gastroenterical Hospital, Saitama, Japan

Present Address:

Y. Sunakawa

Division of Medical Oncology, Department of Medicine,

Showa University Northern Yokohama Hospital, Yokohama, Japan

Present Address:

K. Shimada

Division of Medical Oncology, Department of Medicine, Showa

University Koto-Toyosu Hospital, Tokyo, Japan

Present Address:

N. Katsumata

Department of Medical Oncology, Nippon Medical School

Musashikosugi Hospital, Kawasaki, Japan 
and medically managed. AUC and $\mathrm{C}_{\max }$ increased dosedependently and linear PK profiles were observed. No tumor shrinkage was recorded, but long-term disease stabilization for 25 and 20 months was observed in one patient with clear cell OC $\left(100 \mathrm{mg} / \mathrm{m}^{2}\right)$ and one patient with $\mathrm{GC}\left(400 \mathrm{mg} / \mathrm{m}^{2}\right)$, respectively. No cumulative toxicity occurred in any patient. Farletuzumab was well tolerated in Japanese patients with a similar PK profile as compared with the US population. Long-term disease stabilization was observed in a subpopulation of clear cell OC and GC; both of them were resistant and progressive after standard chemotherapies (ClinicalTrials.gov Identifier: NCT01049061).

Keywords Farletuzumab · Folate receptor $\alpha \cdot$ Monoclonal antibody $\cdot$ Pharmacokinetics $\cdot$ Phase I study

\section{Introduction}

Cancer is the most common and life-threatening disease worldwide whose incidence continues to increase. In Japan, one half of the population has a chance to be diagnosed with cancer in their life time and one third of these Japanese patients lose their lives by cancer, especially lung, gastric, and colorectal cancers $[1,2]$. In addition to surgery or radiotherapy, new medical treatment options including chemotherapy and targeting therapies that are necessary to improve therapeutic outcomes especially in patients with metastatic or recurrent cancer.

Folate plays important roles for DNA synthesis and repair in proliferating cancer cells compared to normal cells [3]. Folate receptor $\alpha$ (FRA) is a protein with high affinity for binding and transporting physiologic levels of folate into cells [4]. High expression of FRA are observed in specific malignant tumors including ovarian cancer, nasopharyngeal epidermoid carcinoma, cervical carcinoma, uterine carcinoma, primary renal cell carcinoma and metastatic pancreatic carcinoma [3]. Protein expression in FRA-positive tumor, nonmucinous epithelial ovarian carcinoma, is associated with tumor progression, and also with platinum-therapy resistance, and poor prognosis [3].

Farletuzumab (MORAb-003; Morphotek, Inc.) is a humanized monoclonal antibody immunoreactive with human FRA [5]. Expression of FRA is identified especially in over $90 \%$ of serous ovarian cancers (OC) [6-8]. Farletuzumab mediates tumor cytotoxicity via antibody-dependent cell cytotoxicity (ADCC) and complement-dependent cytotoxicity (CDC) of an FRA-expressing human OC cell line in vitro [5, 9]. Farletuzumab reduces tumor growth in FRA-expressing OC cells in vivo in a xenograft model $[5,9]$. The first diseaseoriented phase I trial of farletuzumab for OC patients, which was conducted by Konner, reported a promising diseasestabilizing effect and reduction of serum CA125 in patients with heavily pretreated OC [10]. A subsequent phase II study suggests that farletuzumab in combination with carboplatin/ taxane, followed by single-agent farletuzumab maintenance, enhance the response rate and duration of response in platinum-sensitive ovarian cancer patients with first relapse after a remission of 6 to 18 months [11]. However, little information is available as to the anticancer effect of farletuzumab for FRA-expressing non-OC, and no information is reported regarding the relationship between expression level of FRA and antitumor effects $[10,11]$.

We conducted a phase I trial of farletuzumab in Japanese patients with solid tumors and analyzed the relationship between FRA expression level and clinical outcome. This is the first clinical study of farletuzumab that targets not only OC but also FRA-expressing non-OC patients.

\section{Materials and methods}

\section{Trial objectives}

This was a single arm, open-label, dose escalation phase I trial to determine maximum tolerated dose (MTD) by evaluating dose-limiting toxicities (DLTs) as the primary endpoint. Secondary endpoints included investigation of safety and tolerability, estimation of recommended dose (RD) for the next studies, evaluation of antitumor effects, investigation of the pharmacokinetic (PK) profiles of farletuzumab, and detection of human anti-human antibody (HAHA). In addition, a comparison of PK profiles of farletuzumab between Japanese and non-Japanese populations was performed. Progression-free survival (PFS) by FRA expression level was also assessed in subjects whose tumor tissue samples were available. This study was conducted at Saitama Medical University International Medical Center (Hidaka, Saitama) and National Cancer Center Hospital (Tokyo) in Japan.

\section{Patient selection}

Patients who had histologically documented OC regardless of FRA expression were evaluable for the study. Patients with non-OC were also eligible for the study if FRA-expression was confirmed by IHC staining. Patients must have had no standard therapy or had failed prior standard therapies at the time of registration. Other inclusion criteria were as follows: Eastern Cooperative Oncology Group (ECOG) performance status (PS) of 0 or 1 ; age $\geq 20$ and $\leq 80$ years; life expectancy $\geq 12$ weeks; and adequate major organ function (i.e., hemoglobin $\geq 10.0 \mathrm{~g} / \mathrm{dL}$; white blood cell count $\geq 3,000 / \mathrm{mm}^{3}$, $<12,000 / \mathrm{mm}^{3}$; platelet count $\geq 100,000 / \mathrm{mm}^{3}$; total bilirubin $\leq 2.0 \mathrm{mg} / \mathrm{dL}$; alanine transaminase (ALT), aspartate transaminase (AST), and alkaline phosphatase $\leq 2.5$ times upper limit of normal (ULN) or 5 times ULN in the presence of liver metastases; serum creatinine $<2.0 \mathrm{mg} / \mathrm{dL}$ ).

Exclusion criteria were symptomatic metastatic brain tumors; serious and systemic infection requiring medical 
treatment; HIV, hepatitis C antibody, or Hepatitis B surface antigen positive, history of cardiac infarction within 6 months; uncontrolled cardiac disease; liver cirrhosis or interstitial lung disease; active synchronous cancer; history of hypersensitivity to monoclonal antibody or protein formulation; pleural effusion, ascites or pericardial effusion necessary for drainages; and pregnancy or breast feeding.

Drug administration and dose escalation

Single intravenous doses of farletuzumab were administered in cycle 0 on day 1 to monitor the PK profile. From cycle 1 onward, farletuzumab was administered on days 1, 8, 15, and 22 once a week for 4 weeks as one cycle. Farletuzumab was continued weekly, until disease progression or DLT was observed in the patients. DLT was defined as a treatment-related adverse event (AE) that was relevant to any of the following two different criteria: (i) hematologic toxicity, which included grade 4 leucopenia or neutropenia persisting for 7 days or more, $\geq$ grade 3 febrile neutropenia, grade 4 thrombocytopenia, or grade 3 thrombocytopenia requiring blood transfusion; (ii) nonhematologic toxicity $\geq$ grade 3 , excluding abnormal laboratory test results not requiring treatment, nausea, vomiting, and diarrhea.

Farletuzumab was administered by intravenous continuous infusion starting at $1-2 \mathrm{mg} / \mathrm{min}$ and advancing to $5 \mathrm{mg} / \mathrm{min}$ if no AE greater than grade 1 was observed. Premedication for allergic reaction was not allowed before the first dosing. The dose escalation strategy was determined based on a previous phase I study [10] and adapted to a conventional $3+3$ method. The starting dose of farletuzumab was decided to be $50 \mathrm{mg} /$ $\mathrm{m}^{2}$. Dose escalation was followed by 100,200 , and $400 \mathrm{mg} /$ $\mathrm{m}^{2}$ doses. The maximum dose level of $400 \mathrm{mg} / \mathrm{m}^{2}$ was adjusted as in the previous phase I study. If DLT was observed in any planned cohorts within 7 weeks of initiating therapy (cycle 0 and cycle 1 ), up to six patients were to be entered at that dose level. Dose escalation was permitted only after all patients in the cohort had completed cycle 0 and cycle 1 . Intrapatient dose escalation was not permitted.

\section{Safety and efficacy assessment}

Patients were assessed through physical examination, vital signs, complete blood count, chemistries, urinalysis, and ECOG PS. AEs were evaluated at each study visit and graded according to Common Terminology Criteria for Adverse Events (CTCAE) version 3.0 [12]. Immune-mediated AEs including allergic reaction were classified as adverse events of interest based on the judgment by investigators and summarized as infusion related reactions in this paper. Radiological assessment for tumor response was performed at baseline, cycle 1 , and every 8 weeks thereafter. Tumor response was evaluated according to Response Evaluation Criteria in Solid Tumors (RECIST) version 1.0 [13]. PFS was defined as the interval between the first administration of study drug and the first radiographical documentation of disease progression or death from any cause (whichever occurred first). The prior lower dose level below which two or more of six patients experienced DLT was determined to be the maximum tolerated dose (MTD).

\section{Detection of FRA expression}

FRA expression was examined with IHC in patients with solid tumors except for OC. For patients with OC, IHC was performed only for those whose tissue samples were available. Paraffin-embedded samples ( 3 to $5 \mu \mathrm{m}$ slices, at least 3 slices) were placed on glass slides and refrigerated. Frozen samples were transferred into polypropylene tubes to be stored at $-80{ }^{\circ} \mathrm{C}$ until shipment. The collected samples (either paraffin-embedded or frozen) were shipped to the biomarker measurement laboratory (Mitsubishi Chemical Medience, Ibaraki, Japan) on the same day, as feasible. Tumor FRA expression was evaluated using the commercially available anti-FRA antibody, Novocastra Liquid Mouse Monoclonal Antibody Folate Receptor alpha (Leica Biosystems, Newcastle, UK). Pathologists performed grading for staining manually. Immunoreactive intensities were graded as no reaction $(-)$, weak (+: stained light brown with a thin rim along the cell membrane), moderate (++: stained deep-brown with a thin rim along the cell membrane), or strong (+++: stained deep-brown with a thick rim along the cell membrane).

\section{Pharmacokinetic analysis}

Blood sampling for PK analysis was performed for all patients in cycle 0 on day 1 at predose, $30 \mathrm{~min}$ after starting infusion, just after finishing infusion, $30 \mathrm{~min}, 1,2,4,24,72,168,336 \mathrm{~h}$ after infusion. In addition, blood samples at predose in cycle 1 on days $22,29,36$, and 43 and $30 \mathrm{~min}$ after start of infusion, just after finishing infusion, $30 \mathrm{~min}, 1,2,4 \mathrm{~h}$ after infusion on day 43 were also collected.

Serum concentrations of farletuzumab were determined by a validated enzyme-linked immunosorbent assay. Serum farletuzumab levels from 0.313 to $2,000 \mu \mathrm{g} / \mathrm{mL}$ could be determined by this assay. The assay was performed by Catalent Pharma Solutions, LLC (Morrisville, NC). The following PK parameters were calculated by non-compartmental method using WinNonlin ${ }^{\mathrm{TM}}$ Professional (version 6.2.1; Pharsight Corporation Mountain View, CA): maximum observed serum concentration $\left(\mathrm{C}_{\max }\right)$, time at which the highest serum drug concentration occurred $\left(\mathrm{t}_{\max }\right)$, area under the serum concentration-time curve (AUC), terminal phase rate constant $\left(\lambda_{\mathrm{z}}\right)$, terminal elimination half-life $\left(t_{1 / 2}\right)$, and distribution volume at terminal phase $\left(\mathrm{V}_{\mathrm{z}}\right)$. Pharmacokinetic data of a previously reported phase I study [10] was used as reference for comparison of PK profiles between Japanese and US patients. 


\section{Detection of HAHA}

Blood samples for HAHA analysis were collected before farletuzumab administration on day 1 and $336 \mathrm{~h}$ after infusion on day 15 in cycle 0 , preinfusion on days 1 and 15 in cycle 1 , preinfusion on day 1 in cycle 2 and later, at study discontinuation, and at 30 days after the last dose. Analysis was performed by Morphotek, Inc. (Exton, PA). HAHA responses in human serum were detected by a validated electrochemiluminescence (ECL) quasi-quantitative immuno-bridging assay. Farletuzumab was used as the capture antigen, and the biotin-conjugate by virtue of horseradish peroxidase-enzyme conjugation was used as the detection reagent in a bridging assay format. HAHA complexes were subsequently captured on streptavidin-coated microtiter plates and detected in a quasi-quantitative manner via ECL signal generation.

\section{Results}

\section{Patient characteristics}

From December 2009 to March 2011, 16 patients were enrolled in this study and received at least one intravenous infusion of farletuzumab. Patient demographics are listed in Table 1. The median age was 57 years (range, 35-72 years). Eleven patients $(68.8 \%)$ had an ECOG PS score of 0 and five $(31.3 \%)$ had a PS of 1 . Fourteen female patients had OC and two male patients had gastric cancer (GC). All patients had received both surgery and more than two prior chemotherapeutic regimens. Twelve OC patients and 2 GC patents were examined for FRA expression by staining of archival tumor tissue obtained at surgery but not just before farletuzumab treatment. Eleven OC patients $(91.7 \%)$ and 2 GC (100\%) patients were FRA-positive. One patient's OC, deemed FRAnegative, was of unclassified histology. Representative images of immunohistochemical staining for FRA are shown in Fig. 1. Each patient received farletuzumab at one of the dose levels from $50 \mathrm{mg} / \mathrm{m}^{2}$ to $400 \mathrm{mg} / \mathrm{m}^{2}$ according to the dose escalation strategy. Rapid disease progression was observed in one patient (\#13) at the $400 \mathrm{mg} / \mathrm{m}^{2}$ dose level before DLT evaluation and another patient was added to replace this patient.

\section{Safety}

A total of 124 treatment-related AEs (adverse reactions) were reported in the 16 patients during the study. Treatment-related AEs occurring in at least $15 \%$ of patients are shown in Table 2. There were no DLTs in cycle 0 to cycle 1 in the all treatment cohorts. However, all patients experienced at least one adverse reaction during the study. The majority of adverse reactions in this phase I study were grade 1 , and no adverse reactions of grade 3 or higher were observed. There was no clear relationship between dose of farletuzumab and frequency or severity of adverse reactions for the whole study period. No cumulative adverse reactions leading to discontinuation from study treatment were reported. The major adverse reactions were

Table 1 Patient demographics

\begin{tabular}{llllllll}
\hline Patient \# & Dose $\left(\mathrm{mg} / \mathrm{m}^{2}\right)$ & Sex & Age & ECOG PS & Disease & Prior regimens & FRA expression (intensity) \\
\hline$\# 1$ & 50 & Female & 72 & 0 & OC (serous) & 2 & Positive $(+++)$ \\
$\# 2$ & 50 & Female & 66 & 1 & OC (serous) & 3 & Positive $(++)$ \\
$\# 3$ & 50 & Female & 62 & 0 & OC (serous) & 6 & Positive $(+)$ \\
$\# 4$ & 100 & Female & 41 & 0 & OC (clear cell) & 4 & Positive $(+)$ \\
$\# 5$ & 100 & Female & 57 & 0 & OC (serous) & 3 & Positive $(+++)$ \\
$\# 6$ & 100 & Female & 48 & 0 & OC (clear cell) & 2 & Positive $(+)$ \\
$\# 7$ & 200 & Female & 35 & 0 & OC (unclassified) & 3 & Negative $(-)$ \\
$\# 8$ & 200 & Female & 70 & 1 & OC (serous) & 6 & NE \\
$\# 10$ & 200 & Female & 46 & 1 & OC (clear cell) & 3 & NE \\
$\# 11$ & 400 & Male & 71 & 0 & GC (intestinal) & 2 & Positive $(+)$ \\
$\# 12$ & 400 & Female & 50 & 0 & OC (serous) & 4 & Positive $(+++)$ \\
$\# 13$ & 400 & Female & 52 & 0 & OC (serous) & 3 & Positive $(++)$ \\
$\# 14$ & 400 & Female & 58 & 0 & OC (unclassified) & 5 & Positive $(++)$ \\
$\# 15$ & 400 & Female & 48 & 1 & OC (serous) & 4 & Positive $(++)$ \\
\hline 16 & 400 & Female & 57 & 1 & OC (endometrioid) & 7 & Positive $(++)$ \\
\hline
\end{tabular}

ECOG PS Eastern Cooperative Oncology Group performance status; $F R A$ folate receptor $\alpha$; $O C$ ovarian cancer; $G C$ gastric cancer; $N E$ not examined 
Fig. 1 Representative images of immunohistochemical staining for folate receptor $\alpha$. a Patient \#7 (ovarian cancer; negative staining). b Patient \#6 (ovarian cancer; + staining). c Patient \#16 (gastric cancer; + staining). d Patient \#15 (ovarian cancer; ++ staining). e Patient \#1 (ovarian cancer; +++ staining)

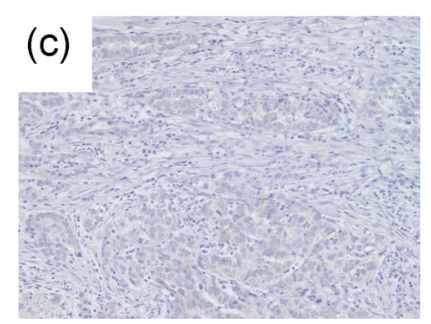

infusion related reaction (15 patients, $93.8 \%$ ), headache (7 patients, $43.8 \%$ ), and nausea and decreased appetite (5 patients each, $31.3 \%$ ). All infusion related reactions reported during the study period occurred during or after the first infusion in cycle 0 . All the reactions developed within $24 \mathrm{~h}$ after the start of farletuzumab administration and resolved within $72 \mathrm{~h}$ after onset. HAHA was not detected in any samples. Although elevation of ALT and AST was observed, all occurrences were classified as grade 1 and were reversible.

\section{Pharmacokinetics}

The mean serum farletuzumab concentration-time profile up to cycle 1 is shown in Fig. 2. Farletuzumab was eliminated biphasically after reaching $\mathrm{C}_{\max }$. Pharmacokinetic parameters on cycle 0 day 1 and on cycle 1 day 22 are shown in Table 3 . The mean $\mathrm{C}_{\max }$ on cycle 0 day 1 ranged from $40.6 \mu \mathrm{g} / \mathrm{mL}$ to $293.4 \mu \mathrm{g} / \mathrm{mL}$, and the mean $\mathrm{AUC}_{(0-\mathrm{t})}$ on cycle 0 day 1 ranged from 4772.7 to $48614.0 \mu \mathrm{g} \bullet \mathrm{h} / \mathrm{mL}$. $\mathrm{C}_{\max }$ and $\mathrm{AUC}_{(0-\mathrm{t})}$ increased almost dose dependently. The mean $\mathrm{t}_{1 / 2}, \mathrm{CL}$, and $\mathrm{V}_{\mathrm{ss}}$ on cycle 0 day 1 were 152.6 to $277.7 \mathrm{~h}, 5.30$ to $10.54 \mathrm{~mL} / \mathrm{h} /$ $\mathrm{m}^{2}$, and 1.87 to $2.29 \mathrm{~L} / \mathrm{m}^{2}$, respectively. These results revealed that farletuzumab was gradually eliminated with a low clearance and a low distribution volume. Meanwhile, the mean $\mathrm{C}_{\max }$ on cycle 1 day 22 ranged from 97.4 to $535.5 \mu \mathrm{g} / \mathrm{mL}$, and the mean $\mathrm{AUC}_{(0-\mathrm{t})}$ and $\mathrm{t}_{1 / 2}$ values on cycle 1 day 22 ranged from 10134.5 to $72055.3 \mu \mathrm{g} \cdot \mathrm{h} / \mathrm{mL}$ and 213.4 to $276.1 \mathrm{~h}$, respectively.

\section{Clinical efficacy}

Of 16 total patients, one patient (\#2) was excluded from analysis of tumor response because no measurable lesion was available. No major tumor shrinkage of partial or complete response was observed in the study. Eight of 15 evaluable patients $(53.3 \%)$ had stable disease, and 7 patients $(46.7 \%)$ had progressive disease as their
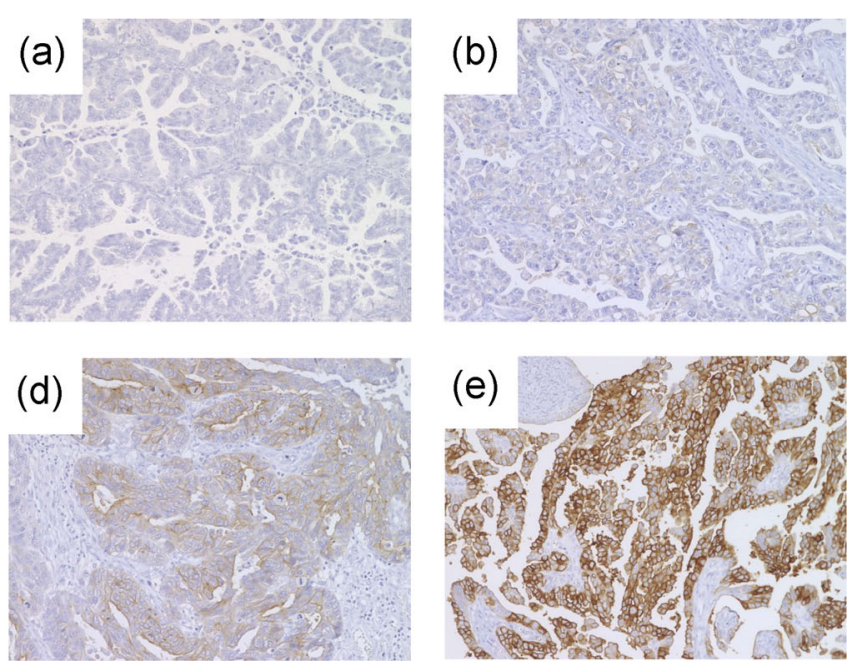

best overall response. PFS for individual patients is shown in Fig. 3. Long-term disease stabilization was observed in one patient (\#6) with clear cell OC treated with $100 \mathrm{mg} / \mathrm{m}^{2}$ for 772 days (25 months) and one patient (\#16) with diffuse type GC treated with $400 \mathrm{mg} / \mathrm{m}^{2}$ for 628 days (20 months). No clear correlations between FRA expression level in archival tumor tissue and PFS were identified in this study; however, the sample size was limited.

\section{Discussion}

Farletuzumab was generally safe and well tolerated in Japanese patients receiving up to $400 \mathrm{mg} / \mathrm{m}^{2}$ doses without encountering any DLTs, and the MTD was not reached at $400 \mathrm{mg} / \mathrm{m}^{2}$ in this phase I study. Adverse reactions evaluated by CTCAE were within grades 1 and 2 and were observed to be dose independent. Common adverse reactions were

Table 2 Treatment-related adverse events and incidence occurring in at least $15 \%$ of patients

\begin{tabular}{llll}
\hline Treatment-related adverse events & $\begin{array}{l}\text { Grade 1 } \\
n(\%)\end{array}$ & $\begin{array}{l}\text { Grade 2 } \\
n(\%)\end{array}$ & $\begin{array}{l}\text { Total } \\
n(\%)\end{array}$ \\
\hline Infusion related reaction & $7(43.8)$ & $8(50.0)$ & $15(93.8)$ \\
Headache & $6^{\mathrm{a}(37.5)}$ & $1(6.3)$ & $7^{\mathrm{a}}(43.8)$ \\
Nausea & $5^{\mathrm{a}}(31.3)$ & 0 & $5^{\mathrm{a}}(31.3)$ \\
Decreased appetite & $5(31.3)$ & 0 & $5(31.3)$ \\
Alanine aminotransferase increased & $4(25.0)$ & 0 & $4(25.0)$ \\
Aspartate aminotransferase increased & $4(25.0)$ & 0 & $4(25.0)$ \\
Fatigue & $4(25.0)$ & 0 & $4(25.0)$ \\
Abdominal pain & $3(18.8)$ & 0 & $3(18.8)$ \\
Blood urine present & $3(18.8)$ & 0 & $3(18.8)$ \\
\hline
\end{tabular}

MedDRA Version 14.1

${ }^{\mathrm{a}}$ One patient is also counted as having an infusion related reaction 
Fig. 2 Mean serum concentrations of farletuzumab after administration up to cycle 1 $\left(n=3\right.$ in 50,100 , and $200 \mathrm{mg} / \mathrm{m}^{2}-$ dose group; $n=7$ in $400 \mathrm{mg} / \mathrm{m}^{2}$ dose group). Error bars show standard deviation

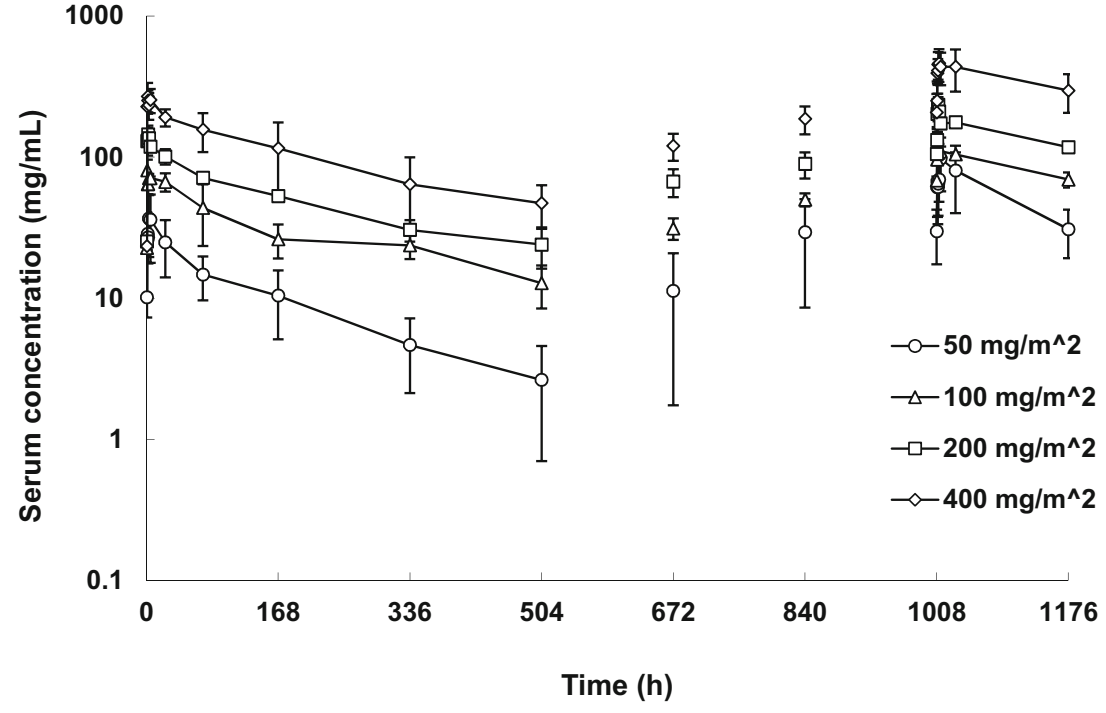

infusion related reaction, headache, nausea, and decreased appetite. These reactions were mild and well tolerated in all patients. Although 15 of 16 patients experienced infusion related reactions such as pyrexia, nausea, chills, and vomiting at first administration of farletuzumab, all of these reactions occurred within $24 \mathrm{~h}$ after the first administration and were managed without discontinuation of farletuzumab therapy. The safety profile of farletuzumab in Japanese patients was similar to that observed in a previous phase I study conducted in the US [10]. Positive HAHA was not detected in any patient in this study.

Pharmacokinetic parameters, including $\mathrm{C}_{\max }$ and $\mathrm{AUC}_{(0-\mathrm{t})}$, increased dose dependently. A low clearance and a low distribution volume characterized the PK profile of farletuzumab, which is similar to the PK profiles reported by other antireceptor antibodies [14]. When the relationship between the

Table 3 Pharmacokinetic parameters after farletuzumab infusion on day 1 of cycle 0 and day 22 of cycle 1

\begin{tabular}{|c|c|c|c|c|}
\hline \multirow[t]{2}{*}{ Parameter } & \multicolumn{4}{|c|}{ Dose of farletuzumab } \\
\hline & $50 \mathrm{mg} / \mathrm{m}^{2}$ & $100 \mathrm{mg} / \mathrm{m}^{2}$ & $200 \mathrm{mg} / \mathrm{m}^{2}$ & $400 \mathrm{mg} / \mathrm{m}^{2}$ \\
\hline Day 1 of cycle 0 & $n=3$ & $n=3$ & $n=3$ & $n=7$ \\
\hline $\mathrm{C}_{\max }(\mu \mathrm{g} / \mathrm{mL})$ & $40.6 \pm 12.3$ & $81.6 \pm 14.4$ & $154.6 \pm 10.6$ & $293.4 \pm 60.8$ \\
\hline $\mathrm{T}_{\max }(\mathrm{h})$ & $2.78 \pm 1.49$ & $2.63 \pm 2.37$ & $3.39 \pm 1.50$ & $4.89 \pm 1.61$ \\
\hline $\operatorname{AUC}_{(0-\mathrm{t})}(\mu \mathrm{g} \bullet \mathrm{h} / \mathrm{mL})$ & $4772.7 \pm 2115.9$ & $14945.5 \pm 2630.4$ & $24060.3 \pm 1788.8$ & $48614.0 \pm 15012.3$ \\
\hline $\operatorname{AUC}_{(0-\text { inf }}(\mu \mathrm{g} \cdot \mathrm{h} / \mathrm{mL})$ & $5420.1 \pm 2648.1$ & $20000.1 \pm 5471.8$ & $34286.8 \pm 7902.0$ & $69704.9 \pm 17583.6^{\mathrm{a}}$ \\
\hline $\mathrm{t}_{1 / 2}(\mathrm{~h})$ & $152.6 \pm 37.3$ & $255.1 \pm 94.5$ & $277.7 \pm 83.5$ & $229.7 \pm 82.0^{\mathrm{a}}$ \\
\hline $\mathrm{CL}\left(\mathrm{mL} / \mathrm{h} / \mathrm{m}^{2}\right)$ & $10.54 \pm 4.03$ & $5.30 \pm 1.65$ & $6.04 \pm 1.34$ & $6.02 \pm 1.35^{\mathrm{a}}$ \\
\hline $\operatorname{Vss}\left(\mathrm{L} / \mathrm{m}^{2}\right)$ & $2.26 \pm 0.81$ & $1.87 \pm 0.27$ & $2.29 \pm 0.20$ & $1.98 \pm 0.55^{\mathrm{a}}$ \\
\hline MRT (h) & $222.5 \pm 52.6$ & $371.2 \pm 109.8$ & $396.6 \pm 123.3$ & $341.0 \pm 113.9^{\mathrm{a}}$ \\
\hline Day 22 of cycle 1 & $n=2$ & $n=3$ & $n=2$ & $n=6$ \\
\hline $\mathrm{C}_{\max }(\mu \mathrm{g} / \mathrm{mL})$ & 97.4 & $119.8 \pm 11.3$ & 238.8 & $535.5 \pm 108.7$ \\
\hline $\mathrm{T}_{\max }(\mathrm{h})$ & 4.56 & $0.99 \pm 0.45$ & 2.22 & $10.36 \pm 10.11$ \\
\hline $\mathrm{AUC}_{(0-\mathrm{t})}(\mu \mathrm{g} \cdot \mathrm{h} / \mathrm{mL})$ & 10134.5 & $14913.9 \pm 1076.4$ & 25555.3 & $72055.3 \pm 22381.4$ \\
\hline $\mathrm{t}_{1 / 2}(\mathrm{~h})$ & - & $249.7 \pm 44.1$ & 276.1 & $213.4 \pm 85.1^{\mathrm{b}}$ \\
\hline
\end{tabular}

Mean \pm SD

$C_{\max }$ maximum observed serum concentration; $T_{\max }$ time at the highest serum drug concentration was observed; $A U C$ area under the serum concentration-time curve; $t_{1 / 2}$ terminal elimination half-life; $C L$ total body clearance; $V_{s s}$ volume of distribution at steady-state; $M R T$ mean residence time ${ }^{\mathrm{a}} n=6$

${ }^{\mathrm{b}} n=3$ 
Fig. 3 Progression-free survival of all patients
FRA

intensity

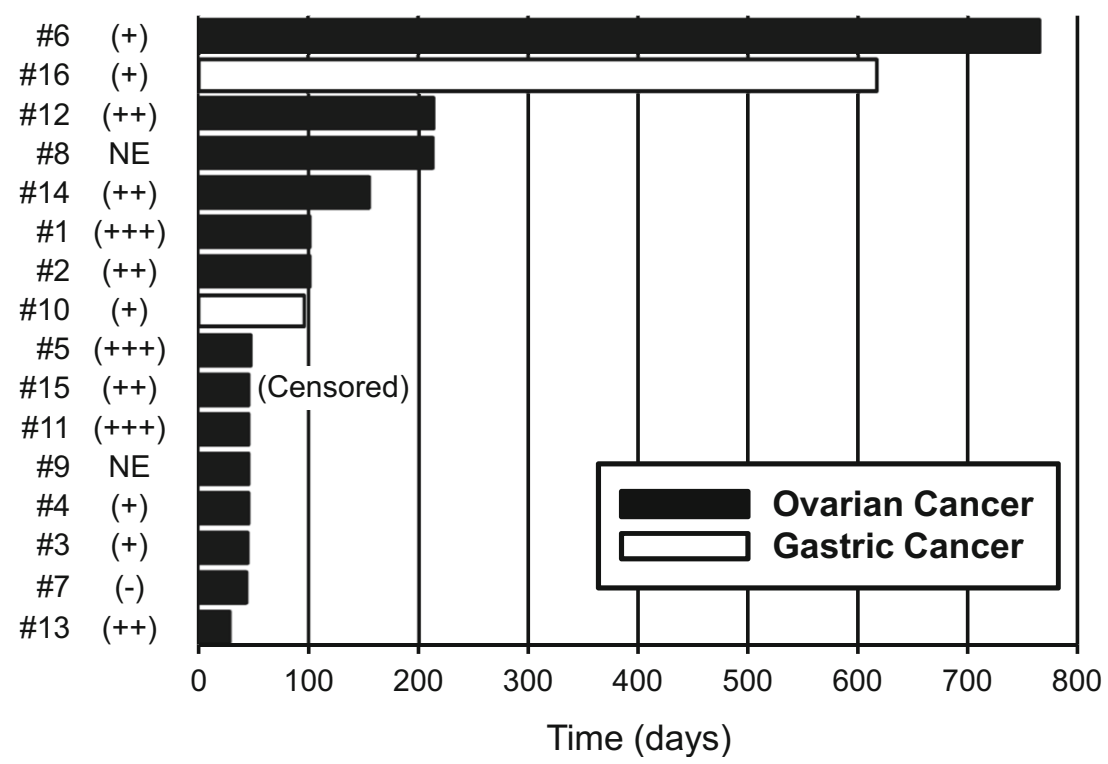

dose and the $\mathrm{PK}$ parameters of $\mathrm{C}_{\max }$ and $\mathrm{AUC}_{(0-\mathrm{t})}$ on cycle 0 day 1 was compared with corresponding parameters observed in the previous US phase I study [10], the comparison revealed similar PK profiles of farletuzumab between Japanese and US patients (Fig. 4). Although the $t_{1 / 2}$ value seems to be different between the Japanese and US studies, the difference can be attributed to the different sampling points (up to 504 and $168 \mathrm{~h}$ after administration for the Japanese and US study, respectively). The serum trough concentrations of farletuzumab after weekly repeated administration at the $50 \mathrm{mg} / \mathrm{m}^{2}$ dose level were more than $10 \mu \mathrm{g} / \mathrm{mL}$, which is beyond the minimum concentration needed to induce ADCC and $\mathrm{CDC}$ in an in vitro pharmacology study [5].

Recently, it was reported in the results of a phase III trial that adding of farletuzumab to standard chemotherapy did not significantly improve PFS in platinum-sensitive recurrent OC [15]. The phase III trial was subsequently conducted using farletuzumab 1.25 and $2.5 \mathrm{mg} / \mathrm{kg}$, equivalent to 50 and $100 \mathrm{mg} / \mathrm{m}^{2}$, respectively, according to the result of a US phase II trial [11]. In our study, we observed long-term stabilization in one patient with clear cell OC treated for 25 months at the dose of $100 \mathrm{mg} / \mathrm{m}^{2}$. Clear cell OC is recognized as a chemoresistant subtype in OC. Actually, this clear cell OC patient was resistant to both paclitaxel/carboplatin and doxorubicin hydrochloride liposome injection. In addition, no DLTs were observed up to $400 \mathrm{mg} / \mathrm{m}^{2}$ of farletuzumab, suggesting that further clinical evaluation of farletuzumab at a higher dose may be warranted. The results of the phase III trial and subset analysis will be published in the near future, and a PK/ pharmacodynamic analysis may provide some information about the recommended dose of farletuzumab in platinum sensitive recurrent OC.
In current study, there was no obvious relationship between the IHC intensity of FRA in archival tumor tissue at surgery and PFS in OC patients, who represent a relatively homogeneous patient background. FRA was only weakly expressed in the clear cell OC patient who demonstrated long PFS (Fig. 1b). FRA expression was also weak in the diffuse type GC patient who also had long-term disease stabilization (Fig. 1c). Although FRA expression in our study requires cautious interpretation because the sample size is small, our finding suggests that higher FRA expression may not correlate with better efficacy of farletuzumab. Cetuximab is an antibody for epidermal growth factor receptor (EGFR), but there is no clear relationship between expression level of EGFR and tumor shrinkage in phase II clinical trial for EGFR expressing colorectal cancer [16]. The clinical significance of FRA expression itself remains unclear in the treatment of farletuzumab. Continued biomarker research in both preclinical and clinical settings including candidate biomarkers such as an ADCC activity is still necessary for evaluation of farletuzumab in enriched patients.

This phase I trial is the first study where farletuzumab was administered to FRA-expressing non-OC patients, and we observed unexpected long-term disease stabilization in one patient with diffuse type GC. Recurrence after surgery was observed in the GC patient during treatment with S-1 (tegafur/ gimeracil/oteracil) as an adjuvant setting, and salvage chemotherapy with two cycles of docetaxel failed. The PFS of the other GC patient was more than 3 months, which is also encouraging for treatment beyond second-line as compared with the results of second-line conventional chemotherapeutic agents $[17,18]$. To best of our knowledge, this is the first report demonstrating the efficacy of farletuzumab in GC. GC 

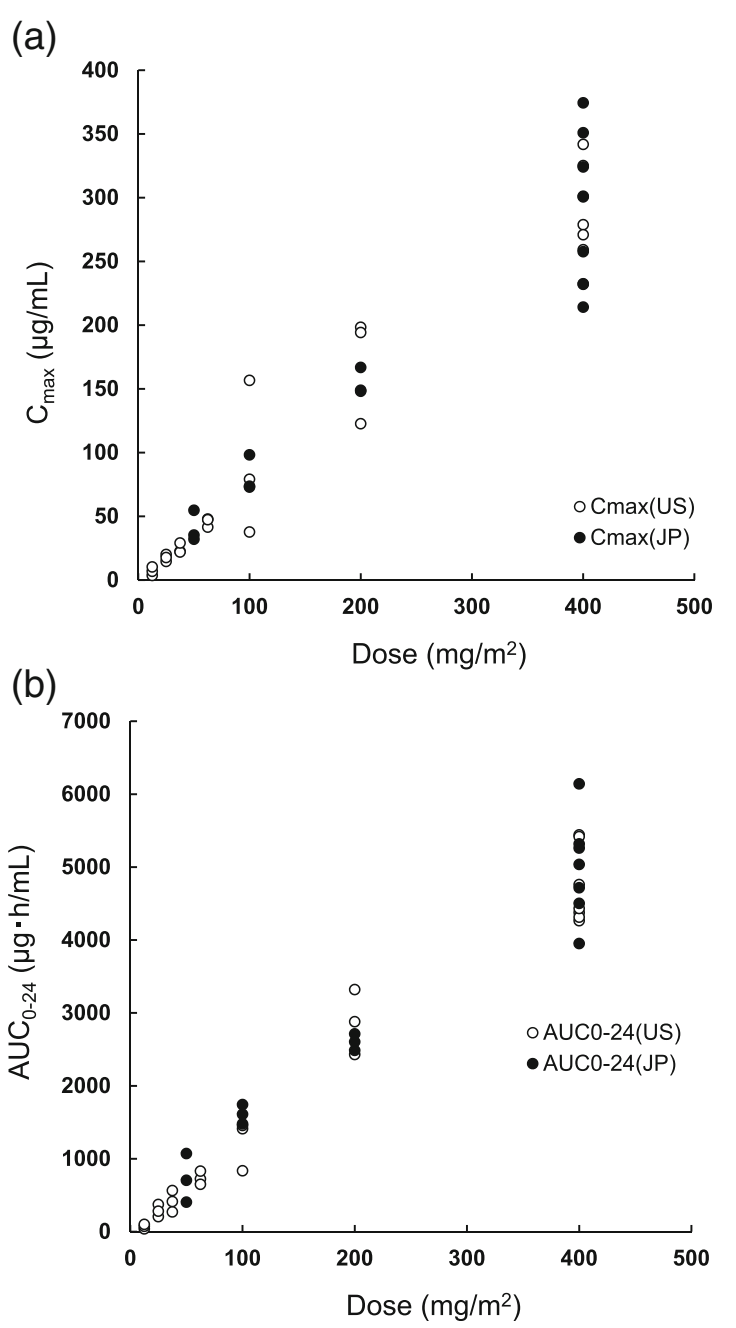

Fig. 4 Relationship between dose and pharmacokinetic parameters of $\mathrm{C}_{\max }(\mathbf{a})$ and $\mathrm{AUC}_{(0-\mathrm{t})}(\mathbf{b})$

is one of the most critical malignancies in East Asian countries including Japan. Both trastuzumab and ramucirumab are identified as an effective targeted agent in the treatment of GC [19, 20], however, further promising targeted drugs are required for prolonging survival. Although a phase III trial in patients with platinum-sensitive recurrent $\mathrm{OC}$ failed to show the efficacy of farletuzumab [15], our findings suggest that GC may be a promising target tumor type for farletuzumab therapy. Further preclinical and clinical evaluation of farletuzumab is warranted in the treatment of GC.

In conclusion, farletuzumab up to a dose of $400 \mathrm{mg} / \mathrm{m}^{2}$ was generally safe and well tolerated in Japanese patients with solid tumors. There were no DLTs and the MTD was not reached up to $400 \mathrm{mg} / \mathrm{m}^{2}$. The safety and PK profiles of farletuzumab were similar between Japanese and US patients. Long-term disease stabilization was observed in a subpopulation of clear cell OC and GC; both of them were resistant and progressive after standard chemotherapies.
Acknowledgments The authors express our thanks to the participating patients and all subinvestigators and research coordinators at two clinical sites. The authors also thank the Eisai study team, especially Nozomu Koyanagi for study management. This study was funded by Eisai Co., Ltd.

Ethics Standards Written informed consent was obtained from all patients before any study-related procedure was performed. Approvals from the institutional review boards were obtained at both Saitama Medical University International Medical Center and National Cancer Center Hospital. This clinical trial was performed in accordance with Japanese Good Clinical Practice (GCP).

Conflict of Interests Yasutsuna Sasaki received lecture fee and research grant from Eisai. Kosei Hasegawa received research grant from Eisai. Keiichi Fujiwara has advisory role for Eisai. Yasuhiro Fujiwara and Noriyuki Katsumata received lecture fee from Eisai. Masayuki Namiki and Minami Matsuda are employed by Eisai. Yutaka Takeuchi is employed by Eisai and owns stock in Eisai. The other authors declare that they have no conflicts of interest.

Open Access This article is distributed under the terms of the Creative Commons Attribution License which permits any use, distribution, and reproduction in any medium, provided the original author(s) and the source are credited.

\section{References}

1. Wakao F, Nishimoto H, Katanoda K, Tsukuma H, Mikami H, eds (2013) Cancer statistics in Japan 2013. Foundation for Promotion of Cancer Research, Tokyo. http://ganjoho.jp/pro/statistics/en/ backnumber/2013_en.html. Accessed 7 Aug 2014

2. Vital statistics in Japan, tabulated by Center for Cancer Control and Information Services, National Cancer Center, Japan. http://ganjoho. jp/pro/statistics/en/table_download.html. Accessed 7 Aug 2014

3. Kelemen LE (2006) The role of folate receptor $\alpha$ in cancer development, progression and treatment: cause, consequence or innocent bystander? Int J Cancer 119:243-250

4. Campbell IG, Jones TA, Foulkes WD, Trowsdale J (1991) Folatebinding protein is a marker for ovarian cancer. Cancer Res 51:53295338

5. Ebel W, Routhier EL, Foley B et al (2007) Preclinical evaluation of MORAb-003, a humanized monoclonal antibody antagonizing folate receptor-alpha. Cancer Immun 7:6-13

6. Garin-Chesa P, Campbell I, Saigo PE, Lewis JL Jr, Old LJ, Rettig WJ (1993) Trophoblast and ovarian cancer antigen LK26. Sensitivity and specificity in immunopathology and molecular identification as a folate-binding protein. Am J Pathol 142:557-567

7. Toffoli G, Cernigoi C, Russo A, Gallo A, Bagnoli M, Boiocchi M (1997) Overexpression of folate binding protein in ovarian cancers. Int J Cancer 74:193-198

8. O'Shannessy DJ, Somers EB, Smale R, Fu YS (2013) Expression of folate receptor- $\alpha$ (FRA) in gynecologic malignancies and its relationship to the tumor type. Int $\mathrm{J}$ Gynecol Pathol 32:258-268

9. Lin J, Spidel JL, Maddage CJ et al (2013) The antitumor activity of the human FOLR1-specific monoclonal antibody, farletuzumab, in an ovarian cancer mouse model is mediated by antibody-dependent cellular cytotoxicity. Cancer Biol Ther 14:1032-1038

10. Konner JA, Bell-McGuinn KM, Sabbatini P et al (2010) Farletuzumab, a humanized monoclonal antibody against folate receptor alpha, in epithelial ovarian cancer: a phase I study. Clin Cancer Res 16:5288-5295 
11. Armstrong DK, White AJ, Weil SC, Phillips M, Coleman RL (2013) Farletuzumab (a monoclonal antibody against folate receptor alpha) in relapsed platinum-sensitive ovarian cancer. Gynecol Oncol 129: $452-458$

12. [No authors listed] (2004) Japanese translation of common terminology criteria for adverse events (CTCAE), and instructions and guidelines. Int J Clin Oncol 9 Suppl III:1-82

13. Therasse P, Arbuck SG, Eisenhauer EA et al (2000) New guidelines to evaluate the response to treatment in solid tumors. European Organization for Research and Treatment of Cancer, National Cancer Institute of the United States, National Cancer Institute of Canada. J Natl Cancer Inst 92:205-216

14. Lobo ED, Hansen RJ, Balthasar JP (2004) Antibody pharmacokinetics and pharmacodynamics. J Pharm Sci 93:2645-2668

15. Vergote IB, Armstrong D, Scambia G et al (2013) Phase 3 doubleblind, placebo-controlled study of weekly farletuzumab with carboplatin/taxane in subjects with platinum-sensitive ovarian cancer in first relapse. Int $J$ Gynecol Cancer 8(Suppl 1):11, 118th International meeting of the European Society of Gynecological Oncology (ESGO), 2013, October 19-22; Liverpool, UK

16. Saltz LB, Meropol NJ, Loehrer PJ Sr, Needle MN, Kopit J, Mayer RJ (2004) Phase II trial of cetuximab in patients with refractory colorectal cancer that expresses the epidermal growth factor receptor. $\mathrm{J}$ Clin Oncol 22:1201-1208

17. Kang JH, Lee SI, Lim Do H et al (2012) Salvage chemotherapy for pretreated gastric cancer: a randomized phase III trial comparing chemotherapy plus best supportive care with best supportive care alone. J Clin Oncol 30:1513-1518

18. Hironaka S, Ueda S, Yasui H et al (2013) Randomized, open-label, phase III study comparing irinotecan with paclitaxel in patients with advanced gastric cancer without severe peritoneal metastasis after failure of prior combination chemotherapy using fluoropyrimidine plus platinum: WJOG 4007 trial. J Clin Oncol 31:4438-4444

19. Bang YJ, Van Cutsem E, Feyereislova A et al (2010) Trastuzumab in combination with chemotherapy versus chemotherapy alone for treatment of HER2-positive advanced gastric or gastro-oesophageal junction cancer (ToGA): a phase 3, open-label, randomised controlled trial. Lancet 376:687-697

20. Fuchs CS, Tomasek J, Yong CJ et al (2014) Ramucirumab monotherapy for previously treated advanced gastric or gastrooesophageal junction adenocarcinoma (REGARD): an international, randomised, multicentre, placebo-controlled, phase 3 trial. Lancet 383:31-39 